\title{
Miranda
}

Revue pluridisciplinaire du monde anglophone /

Multidisciplinary peer-reviewed journal on the English-

speaking world

$19 \mid 2019$

Rethinking Laughter in Contemporary Anglophone Theatre

\section{Tennessee Williams in translation : retour sur la première traduction en français de Camino Real}

Retours d'expérience

\section{Bertrand Augier}

\section{(2) OpenEdition}

\section{Journals}

Édition électronique

URL : http://journals.openedition.org/miranda/21216

DOI : $10.4000 /$ miranda.21216

ISSN : 2108-6559

Éditeur

Université Toulouse - Jean Jaurès

Édition imprimée

Date de publication : 7 octobre 2019

Référence électronique

Bertrand Augier, «Tennessee Williams in translation : retour sur la première traduction en français de Camino Real », Miranda [En ligne], 19 | 2019, mis en ligne le 11 octobre 2019, consulté le 16 février 2021. URL : http://journals.openedition.org/miranda/21216; DOI : https://doi.org/10.4000/miranda. 21216

Ce document a été généré automatiquement le 16 février 2021.

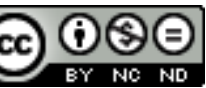

Miranda is licensed under a Creative Commons Attribution-NonCommercial-NoDerivatives 4.0 International License. 
Tennessee Williams in translation : retour sur la première traduction en français de Camino Real

Retours d'expérience

Bertrand Augier

Fig 1

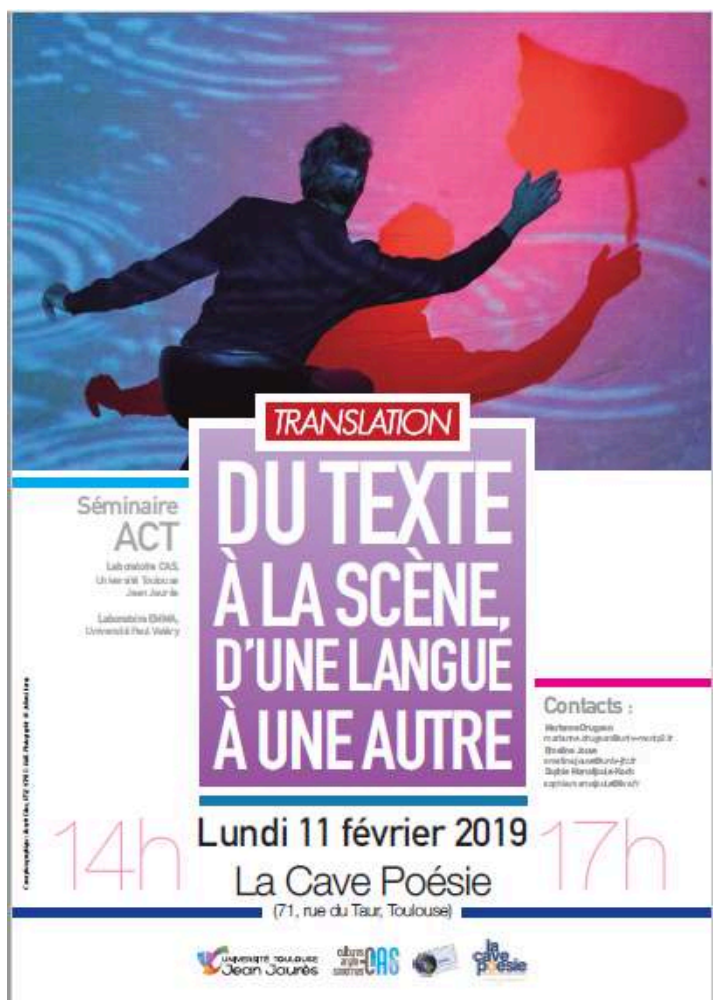

Séminaire ACT, 11.02.2019

Crédit : Benoît Colas. 
Fig 2

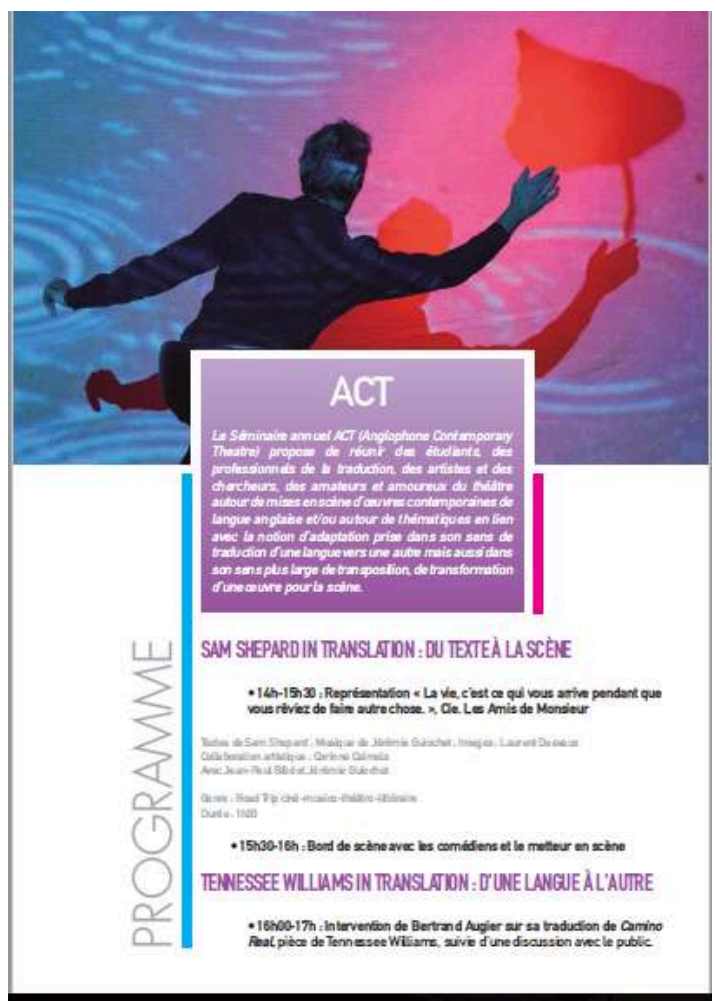

Séminaire ACT, 11.02.2019, programme

Crédit : Benoît Colas.

\section{Préambule}

1 Traduire. Que cela implique-t-il en termes de maîtrise des langues concernées, langue de départ et langue d'arrivée ? Une maîtrise parfaite de la langue de départ, si elle est bien sûr un atout, n'est pas un must absolu: ce qu'il faut bien davantage, c'est la connaître, via les études, la pratique ou la lecture, pour savoir comment l'aborder; la connaître pour savoir comment la faire dialoguer avec le français - car c'est bien de dialogue qu'il s'agit, le dialogue que le traducteur noue entre deux univers linguistiques pour transporter intact et vivant d'une langue à l'autre tout un univers narratif, émotionnel et esthétique : celui de l'auteur.

2 En revanche, s'il y a un must absolu, c'est bien sûr la maîtrise de la langue d'arrivée, la langue française; et même, avant sa maîtrise, bien plus important, car de là naît la justesse : le goût, l'amour de la langue, la sensibilité à la langue.

3 Si donc la traduction littéraire comporte bien entendu une dimension technique, cela reste - et doit rester - radicalement et avant tout un acte artistique, avec toutes les exigences qui vont avec, et notamment en matière de probité et de sincérité. La dimension à dominante souvent technique de ce qui suit ne doit pas le faire oublier. 


\section{Objectifs de la présentation}

4 Le séminaire dans le cadre duquel a été conçue cette présentation s'intitule "Translation : du texte à la scène, d'une langue à l'autre ».

5 Si comme dit Molière, "le théâtre n'est fait que pour être vu » (ou joué), il faut bien commencer par l'écrire. Par cette action en deux temps, le théâtre est comme une gravure à l'eau-forte ${ }^{1}$, mais réalisée à plusieurs : l'auteur, le dramaturge, prépare la plaque de métal, l'enduit de vernis, grave... mais ne la trempe pas lui-même dans le bain d'acide qui va décider de la profondeur des creux et du tranchant des arrêtes; plutôt, il remet la plaque ainsi gravée à ceux qui vont jouer la pièce, metteur en scène et comédiens, à qui il revient d'assumer les dernières étapes : s'emparer de la plaque, se l'approprier et la tremper autant que nécessaire dans leur acide à eux, l'acide très particulier de leur sensibilité, d'où elle ressortira sous la forme vivante, colorée et sonore de la pièce jouée.

6 Et ce qui est vrai de l'écriture est également vrai de la traduction.

7 Cette présentation va donc se concentrer sur cette plaque et sur la manière de la préparer et de la travailler, de la graver, pour pouvoir la remettre au metteur en scène et aux comédiens (ou au lecteur - et c'est vrai bien entendu aussi de la prose, tant chaque lecteur est au fond de lui-même le scénographe en chef de tout ce qu'il lit) et leur ouvrir ainsi dans les meilleures conditions les portes du jardin de l'auteur, et quel jardin, s'agissant de Tennessee Williams! Et quel encore plus extraordinaire jardin s'agissant de Camino Real, ce jardin où la violette peut être plus forte que le rocher !

8 Ce titre, faut-il le prononcer caMIno reAL, "à l'espagnole", ou plutôt, selon les instructions de Williams, dans sa prononciation «à l'anglaise », CAmino REal (où l'en entend $\left.r^{-\prime} ə l\right)$ ? Doit-on y entendre une évocation de quelque voie « royale », ou plutôt d'un monde où domine et s'impose le « réel »?

9 La première difficulté de traduction surgit donc dès le titre. Car si en anglais la prononciation requise permet de créer la possibilité d'un premier glissement sémantique, cela est inopérant en français où l'on prononce assez naturellement «à l'espagnole », et où « real » ne signifie en rien « réel ».

Ceci est assez emblématique de la difficulté permanente de l'acte de traduction, difficulté qui tient à ce que l'on pourrait appeler la transposition et le dosage de la richesse, avec ici un titre anglais qui est plus riche que le titre français.

Et c'est assez emblématique aussi de ce qui arrive parfois : la nécessité de renoncer, car ici, trouver un autre titre aurait certainement été perdre plus que gagner. Mais nous y reviendrons.

Pour en venir au fond de cette présentation, abordons maintenant la traduction de Camino Real en évoquant successivement :

Dans une première partie assez courte : le travail préparatoire - la plaque, le vernis ;

Dans une seconde partie, le travail de traduction proprement dit - la gravure. 


\section{Le travail préparatoire}

\section{Avec dire?
dire}

Aucune traduction littéraire n'est facile. Mais certaines le sont plus que d'autres, et d'autres moins. À tel point que d'après certains, il y a même des textes qui seraient impossibles à traduire : typiquement, la poésie.

Dans tous les cas de figure, il existe un élément absolument déterminant qui doit être impérativement pris en compte dès le tout début du travail de traduction : l'intention de l'auteur. Si celle-ci est généralement limpide dans un texte didactique ou scientifique, il en va tout autrement dans un texte littéraire.

Dans une non-fiction, rien n'existe que ce qui est dit, exprimé. Dans un texte de fiction, c'est presque tout l'inverse... et c'est bien plus compliqué.

Dans un texte de non-fiction, mots et phrases n'ont qu'un rôle : encapsuler, pour ainsi dire, un sens, aussi fermement et précisément que possible, pour le transmettre sans déperdition et sans déformation ni altération, semblablement à la gélule qui, dans un médicament, contient le principe actif.

Dans un texte de fiction, mots et phrases n'ont qu'un rôle : suggérer quelque chose de bien plus grand qu'eux-mêmes, libérer l'imaginaire et faire naître l'immatériel absolu : l'émotion.

Et pour traduire ce «faire naître l'émotion », il faut savoir ce que voulait l'auteur, déchiffrer son intention.

Là est la difficulté. Un texte comme Camino Real n'est pas immédiatement, totalement figuratif. Ce n'est pas une nature morte, avec une pomme sur un plateau.

Faire face à cette difficulté impose de distinguer deux niveaux d'intention, tout simplement parce qu'il va falloir y associer deux niveaux de préparation : un niveau général, et un niveau particulier.

\section{La préparation de niveau général}

La première chose à faire, c'est de lever le nez de son livre et de s'enrichir des réponses qu'ont déjà pu élaborer tous ceux - auteur compris - qui se sont déjà posé la question. Internet, naturellement, sous réserve de faire preuve sans relâche d'esprit critique, offre une intéressante source d'informations.

Parmi des sources souvent nombreuses et d'intérêt très variable, il pourra arriver que l'on identifie des documents d'une exceptionnelle richesse, comme la thèse sur le théâtre de Tennessee Williams, de Sophie Maruéjouls-Koch, enseignant-chercheur à l'Université de Toulouse Jean Jaurès², parfait exemple de document des plus précieux pour le traducteur.

26 La deuxième chose à faire, et quel que soit ce qui a pu être glané sur internet, c'est de lire tout le texte avant de se lancer. Cela peut paraître une évidence, mais cela peut n'être pas sans risque : n'est-ce pas là contraire à l'esprit même d'une pièce ou d'un roman qui «avance» et se dévoile peu à peu? Ne faut-il pas rester dans la même logique de découverte de ce qui arrive, logique qui est celle du spectateur ou du lecteur 
lambda, voire de l'auteur? À trop préparer, analyser, rationaliser, ne prend-on pas le risque de privilégier une approche technique du texte, au détriment de sa composante sensible? De gommer certaines des intentions de l'auteur, qui souvent dissémine ici et là des leurres, des fausses pistes, des flous que le traducteur doit pleinement respecter et relayer / transposer, sous peine d'affaiblir, affadir et trahir le texte ? Et ce plein respect n'est-il pas en risque dès lors qu'à l'innocente ignorance du premier regard succèdent les fausses spontanéités du raisonnement et de l'analyse?

Ce risque existe certainement. Mais a contrario une telle "pré-lecture» permet au traducteur de prendre la mesure du texte et d'en faire une reconnaissance approfondie, comme un sportif va reconnaître le tracé de sa course du lendemain, soucieux qu'il est d'éviter de prendre de mauvais embranchements, de faire de mauvais choix.

Ici, il s'agit de lire non pas en mode analytique - ce sera pour plus tard -, mais en mode « lecteur ", juste pour faire naître « comme d'habitude » ses émotions de lecteur, et faire opérer la première cristallisation de ces émotions, d'où va naître à son tour l'idée que vous vous faites de l'intention de l'auteur.

Cette première lecture doit permettre notamment de réaliser un premier repérage :

D'une part, des relations entre événements et personnages en les inscrivant dans les dimensions temporelle et spatiale du texte et de la scène.

Application dans Camino Real - S'agissant d'une pièce très animée, il n'est pas inutile de faire un plan de scène et des déplacements, cela permet de donner une assise concrète à des événements par ailleurs assez décousus.

D'autre part, des éventuels stratagèmes de l'auteur: leurres, fausses pistes, flous et sauts spatiaux ou temporels, qui constituent des éléments de structure du schéma narratif ou dramatique.

33 Application dans Camino Real - Dans cette pièce, qui fait la part belle à la spontanéité débridée de personnages souvent décalés et exubérants, Tennessee Williams ne recourt pas à de tels stratagèmes. Une autre pièce de Tennessee Williams, Vieux Carré, permet d'illustrer toutefois ce point, avec l'exemple du personnage très particulier de l'Écrivain, qui cumule un rôle de personnage actif, directement partie prenante à l'action de la pièce, avec un rôle de personnage-narrateur : la bonne compréhension de cette dualité est un élément déterminant pour bien saisir quelles étaient les intentions de l'auteur lorsqu'il écrivit Vieux Carré, et une traduction sensible des passages impliquant cet Écrivain-acteur-narrateur impose que le traducteur ait acquis une certaine intimité avec lui, ce que seule une lecture complète de la pièce peut permettre.

Enfin, des répétitions, échos, renvois et autres variations de registres (de l'humoristique au pathétique, du grandiloquent au superficiel), ainsi qu'une validation a posteriori de certaines "options de compréhension" via la rétro-perspective que le traducteur peut dégager à la fin de la lecture.

Application dans Camino Real - Plusieurs codes répétitifs accompagnent le lecteur / spectateur tout au long de la pièce : le piping des Nettoyeurs de Rue, le Little white ship to sail the dangerous night in de A. Ratt, et il est impératif de clairement retrouver dans le texte d'arrivée ce type de stratagèmes d'auteur qu'une première lecture complète et d'une traite peut permettre de ressentir et détecter.

Cette première lecture doit notamment aussi permettre d'anticiper certains effets de « rétro-action » potentiellement fâcheux. 
Il en va ainsi, par exemple, de la répétition d'un terme ou d'une expression, écho qui revient plusieurs fois dans un texte, et dont la traduction se trouve pour ainsi dire " contrainte " par le texte environnant à un certain endroit et pas à d'autres : lorsqu'on arrive à l'endroit concerné du texte de départ, le fait que la traduction se trouve ainsi " contrainte » rétro-agit sur les occurrences précédentes, qu'il faut reprendre bien sûr pour conserver l'homogénéité de la traduction, et cela peut avoir sur le reste du texte des « effets domino » assez pénalisants.

Application dans Camino Real - Dans ses premières occurrences, il aurait pu paraître pertinent de traduire Skid Row (le quartier déshérité occupant un côté de la scène, par opposition au côté cossu représenté, de l'autre côté, par l'hôtel Siete Mares) par l'image figurée de "Cour des miracles", mais si cela aurait bien fonctionné tant que l'on était dans le descriptif et les didascalies, il en aurait été différemment au Bloc 14, où mettre dans la bouche de Kilroy une évocation de ces « chambres de la Cour des miracles, où l'on dort pour un dollar » aurait sonné de manière très malheureuse en associant des éléments aux arrière-plans incompatibles (Kilroy et Cour des miracles, Cour des miracles et dollar). Mieux valait donc conserver tel quel «Skid Row », et mieux valait en avoir l'idée dès le démarrage de la traduction, afin d'éviter d'avoir à reprendre toutes les occurrences précédentes, et de devoir s'assurer que cette reprise elle-même ne venait pas bousculer les passages concernés.

Plus l'auteur joue de ces répétitions, échos et autres métaphores filées, plus il est nécessaire de procéder avec soin à cette reconnaissance générale.

\section{La préparation de niveau particulier}

40 Le niveau particulier porte sur le texte en tant que sous-ensembles: chapitres, paragraphes, phrases.

1 Le point précédemment évoqué est également valide ici : il faut lire «à l'avance» chapitres et paragraphes; ce n'est que comme cela que l'on peut suffisamment analyser le texte pour y déterminer les éléments de structure - s'il y en a -, les éléments stylistiques et les jeux de mots et de sonorités qu'il est souhaitable, pour ne pas dire impératif, de reprendre.

Le message ici est parfaitement clair : il ne faut pas se concentrer que sur le sens, la forme est aussi importante et il faut être d'une vigilance intense et permanente pour ne pas laisser passer l'élément de forme « qui compte » et dont il faut rendre compte.

Mais ceci n'est pas toujours possible.

Application dans Camino Real - Voici un exemple, ou plutôt un contre-exemple : Bloc 12, la Gitane dit à Kilroy, « No one is gypped at the Gypsie's », que le traducteur a rendu par «Personne ne se fait arnaquer chez la Gitane»; si le sens est correct, cette traduction échoue à rendre la belle et sonore assonance gypped / Gypsie's pour laquelle aucun équivalent n'a été trouvé malgré d'intenses recherches - il en va ici comme avec le titre : parfois, il n'y a pas d'alternative au renoncement.

À l'issue de cette première et rapide partie, deux messages principaux se dégagent qu'il est important de bien garder à l'esprit :

D'une part, traduire est un exercice d'humilité : l'intention générale et les intentions particulières de l'auteur doivent passer avant toute ambition personnelle du traducteur, 
et il faut toujours se garder de conclure trop vite et de s'imaginer avoir capté les intentions de l'auteur dans toute leur complexité et leur finesse.

Ce qui, d'autre part, impose d'autant plus de faire un véritable leitmotiv, une devise absolue, des questions qui suivent, questions qui doivent sans cesse pousser le traducteur à se remettre en cause : quelles étaient les intentions de l'auteur? En tant que traducteur, ai-je fait l'effort suffisant pour les identifier ? Y suis-je fidèle?

\section{La réalisation}

La plaque de métal et son vernis étant maintenant prêts, il est possible d'entrer un peu plus dans le détail pratique de la traduction de Camino Real: la gravure proprement dite.

Les points suivants seront successivement abordés :

Les noms propres;

La localisation ;

Morphologie des mots, mots déformés et tronqués;

Les mots en langue étrangère ;

Jeux sémantiques et jeux de sonorités ;

Figures de style et « enrichissement abusif »;

Gestion des « béquilles » (ce qui permet de «faire tenir debout » le texte).

\section{Les noms propres}

51 Dans la littérature comme ailleurs (au cinéma par exemple), il est des noms propres que l'on ne traduit pas - Superman, David Copperfield - et d'autres que l'on traduit - les noms de lieux dans Le Seigneur des Anneaux de J. R. R. Tolkien, par exemple, avec ces étonnants «Cul-de-Sac », "Creux-de-Crique » ou "Chateaubouc », qui cohabitent avec d'autres noms qui, eux, demeurent inchangés, tel le sinistre « Mordor ».

Camino Real n'échappe pas au questionnement, du moins pour les noms susceptibles de véhiculer un sens en sus de la seule désignation d'un personnage ou d'une chose, ou qui n'ont pas d'existence antérieure propre - bien sûr il est hors de question de traduire Casanova par "Maisonneuve", et bien sûr on ne se posera pas la question de la transposition pour les autres " célébrités » avérées, qu'elles soient réelles ou de fiction (Charlus, Byron, Kilroy et autres).

Parmi les autres noms propres, il en est qui peuvent demeurer tels quels car ils sont pour le lecteur / spectateur français suffisamment évocateurs et clairs «en l'état»: Nursie, le Fugitivo.

D'autres en revanche s'avèrent plus problématiques : le titre même de la pièce, Camino Real, déjà évoqué, mais pas seulement. Il en va également ainsi de :

Loan Shark, le prêteur sur gages : le nom est signifiant, mais intraduisible sans friser le ridicule.

Le contournement pour le lecteur se fait via la liste des personnages: ajout d'une mention « [Loan Shark], le prêteur sur gage », et via les didascalies : dans l'exposition du prologue, ajout de la mention, « [Chez Loan Shark], du nom de l'usurier ». 

traduire un nom propre il convient également de prendre en compte le confort des " utilisateurs ", c'est-à-dire le confort des lecteurs mais aussi, s'agissant d'une pièce de théâtre, le confort des comédiens. La solution envisagée par le traducteur est-elle de nature à poser une difficulté potentielle pour les comédiens? Loan Shark et Skid Row coulent assez bien dans une diction française; Ritz Men Only moins bien peut-être... C'est là au traducteur qu'il revient de bien doser sa prise en compte des différents paramètres.

68 C'est ainsi que l'on a, dans ce Bloc 4, en l'espace de huit lignes, trois options de traduction qui se côtoient - traduction littérale, traduction « en substance » et... nontraduction avec solution de contournement.

69 Sur le fond, cette mosaïque d'approches, ce patchwork pourrait-on dire, sont-ils à un titre ou à un autre gênants? Non, sera-t-il possible de répondre, ou du moins sans qu'en résulte un quelconque préjudice sensible. Mais c'est là une situation qui illustre 
bien le fait que dès lors que l'on a affaire à du non-dit, du suggéré, de l'immatériel émotionnel ou imaginaire - tous éléments indissociables de l'essence même d'un "texte littéraire»-, le texte d'arrivée ne traduit jamais à 100\% l'ensemble des dimensions d'un texte de départ, l'objectif le plus ambitieux ne pouvant viser mieux qu'entre 90 et $99 \%$

\section{La localisation} « mètres ". here ");

Il s'agit là d'un des aspects les plus délicats à traiter car c'est un de ceux où finalement il $\mathrm{y}$ a le moins de règles et où il est le plus question de jugement: entre le texte (et l'intention) d'origine et le lecteur / spectateur de destination, où le traducteur doit-il mettre le curseur pour « optimiser », pourrait-on dire, le point de rencontre?

\section{Parfois c'est extrêmement facile.}

Application dans Camino Real - Dans l'exposition du prologue, Sancho suit Don Quichotte «a couple of yards behind»: peu d'hésitation avant de traduire ce «yards» par

\section{Parfois c'est un peu plus compliqué.}

Applications dans Camino Real - La fin du Bloc 2 voit l'entrée en scène de Kilroy, qui s'arrête devant un graffiti où est écrit "Kilroy is coming ", qu'il transforme en " Kilroy is here $»^{3}$. Faut-il traduire, ou pas, ces graffitis ?

Certains arguments militent en faveur d'une traduction : une version française est bien sûr plus intelligible pour les lecteurs / spectateurs non anglophones, et elle résonne parfaitement avec la reprise « orale» du graffiti, notamment en fin de Bloc 14 lorsque Kilroy apostrophe les Nettoyeurs de Rue en leur lançant un «Kilroy is here» nécessairement traduit par « Kilroy est là »;

D'autres arguments vont dans le sens de la conservation de la version anglaise du texte de départ : d'une part le maintien du côté « historique » du graffiti (s'il est connu des lecteurs / spectateurs, c'est de façon quasi-certaine dans sa version originale, et s'il n'est pas connu, il n'évoque rien de toute façon), et d'autre part le retour au graffiti à la fin du seizième et dernier Bloc, car même si les modifications successives apportées par Kilroy au graffiti n'auraient pas posé de difficultés de scénographie dans une version

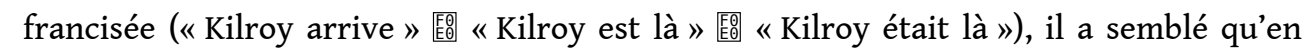
langue d'origine l'enchaînement était plus fluide et d'une meilleure "sonorité

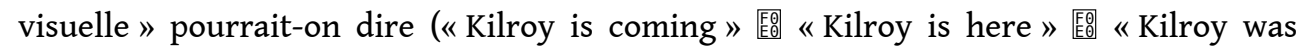

La solution retenue a consisté à... renoncer à la corrélation stricte entre le graffiti écrit et l'expression verbale qu'en fait Kilroy Bloc 14. Conserver la version anglaise du graffiti écrit («Kilroy is here ») en la découplant de sa version orale («Kilroy est là ») n'a pas paru amoindrir la version d'origine ni créer avec elle de rupture significative, le caractère bilingue du traitement retenu ne pouvant guère provoquer quelque gêne que ce soit chez le lecteur / spectateur.

78 Bloc 4, Loan Shark évoque «a piece of green paper with Alexander Hamilton's picture on it ", c'est-à-dire un billet de 10 dollars. Faut-il transposer cette périphrase, par exemple en "Pascal», ou en "Delacroix», noms alternatifs par lesquels étaient désignés les anciens billets français de 500 et 100 francs? 
79 Non car cela «localiserait» excessivement, dénaturerait le texte, et au regard des besoins prioritaires de cohérence, si l'on peut dire, poserait plus de problèmes que n'apporterait de bénéfices (du fait par exemple de la précision donnée par Loan Shark quant à la couleur verte du billet, couleur qui n'était pas celle des billets français, mention qui par ailleurs constitue pour le lecteur / spectateur français un indice fort, tant est courant l'emploi de la périphrase « billet vert » pour évoquer génériquement le dollar).

On l'a vu pour les noms propres, l'ajout limité d'informations peut permettre aussi de contourner «discrètement » les difficultés de localisation. Que faire, Bloc 4, dans une réplique de Kilroy, de l'évocation de ce qu'il nomme de manière très minimaliste «le $\mathrm{Y} »$ ?

81 L'ajout de "YMCA » deux répliques plus loin, lorsque Kilroy explique ce qu'est le « $\mathrm{Y}$ » au Baron de Charlus (qui, en bon personnage français, ignore naturellement de quoi il s'agit), permet de mettre le lecteur / spectateur sur la voie et ainsi d'éviter (en partie seulement peut-être) que le texte demeure strictement incompréhensible pour le plus grand nombre.

Parfois, il faut savoir "régresser ", pourrait-on dire, pour venir à quelque chose de compréhensible. Ainsi Bloc 6, comment traduire «Greyhound depot » et «Greyhound bus depot »?

83 Si Greyhound est le nom d'un grand opérateur de lignes de cars universellement connu aux États-Unis, il n'en est pas de même en France, et il semble préférable, plutôt que maintenir cette référence précise mais peu pertinente pour le lecteur / spectateur français, d'abandonner le nom propre Greyhound pour glisser vers un plus simple et plus immédiatement parlant « gare routière ».

Se pose également la question des références culturelles sans équivalent direct, et pour lesquelles il faut parfois, et selon les cas :

Se montrer un peu inventif : ainsi Bloc 12, "Screen Secrets ", titre réel d'un magazine de cinéma américain, qu'il n'est pas pertinent de conserver du fait du risque élevé de non-compréhension par le lecteur / spectateur français, et que le traducteur a rendu « en substance » par « Les coulisses du cinéma »;

Savoir renoncer et s'appuyer sur d'autres éléments de scénographie ou de décor, parce que la transposition directe est parfois simplement impossible : il en va ainsi des trois boules de cuivre, enseigne du prêteur sur gages communément reconnue aux ÉtatsUnis et dans plusieurs autres pays, mais pas en France, avec pour solutions de contournement possibles, pour le lecteur, des ajouts descriptifs ou de didascalies, et pour le spectateur, des éléments de décor (une mention « Prêteur sur gages » sur la vitrine de Loan Shark, celle-là même que surmonte l'enseigne aux trois boules de cuivre, par exemple) ou de scénographie (une gestuelle appropriée, lorsque c'est par synecdoque (" trois boules de cuivre ») que Kilroy évoque le prêteur sur gages (Bloc 3)).

Doivent aussi être traitées les expressions purement idiomatiques, également dépourvues d'équivalent direct :

Ainsi Bloc 12, le «Skiddoo! » de la Gitane, sans équivalent français, et que le traducteur a rendu par « On met les voiles!»

Une autre difficulté tient aux références à des personnalités connues de l'époque et du lieu, sans équivalent dans le référentiel des lecteurs / spectateurs français : 
90 Ainsi Bloc 12, avec l'évocation de «Winchell», nom d'un journaliste américain spécialiste du scooping à la fin des années 50, non transposable dans l'univers français, que l'on a conservé mais agrémenté d'une note pour le lecteur, sachant que demeure pour le spectateur une " petite bulle de mystère »;

91 De même un peu plus loin dans le même Bloc 12, avec la mention de "Walgreen ", chaîne de pharmacies fondée à Chicago en 1901 et présente sur tout le territoire des États-Unis : il paraîtrait inapproprié de traduire « I gotta go down to Walgreen's » par " il faut que j'aille chez Carrefour », de même que, si comme on l'a vu il est parfois possible d'instiller de-ci de-là une petite information, il a paru, dans le dialogue extrêmement rythmé de ce Bloc 12 où Walgreen est cité, inapproprié d'alourdir et ralentir le texte par l'ajout d'un «[Il faut que j'aille] au magasin du coin de la rue [chez Walgreen faire de la monnaie"].

Encore plus délicates à traiter sont les références personnelles de l'auteur accessoirement parce que l'on n'est pas absolument certain que ces mêmes références aient déjà été très parlantes pour les spectateurs lambda de l'époque :

93 Ainsi, Bloc 12 toujours, "Todd's place ", qui évoque semble-t-il un hôtel où Tennessee Williams résida durant son séjour à Acapulco, en septembre $1940^{4}$, reste impossible à transposer... que l'on traduit donc par un simple « Chez Todd».

Un dernier exemple avec les nombreuses chansons du Clochard qui agrémentent le déroulé du texte: faut-il traduire le texte des paroles proposées par l'auteur, ce qui impose de parvenir en même temps à en conserver le sens et à rester dans la mélodie d'origine, alors même que l'on ne dispose pas, ou peu, de l'arrière-plan culturel correspondant?

Le choix de traduire a paru s'imposer dans cette première version française de Camino Real, afin d'éviter que ces séquences ne soient dans le texte d'arrivée purement « musicales » là où, dans le texte de départ, elles sont elles-mêmes porteuses de sens.

\section{Morphologie des mots, mots déformés et tronqués}

96 Il s'agit là d'une zone récurrente de difficulté pour le traducteur, même si Camino Real n'est pas dans ce domaine un titre d'une particulière complexité. C'est un sujet compliqué à gérer parce que, si dans l'écrit en langue anglaise déformer les mots, les tronquer, les noter en mode phonétique, est quelque chose d'admis, de courant et d'assez naturel, il n'en va pas de même dans l'écrit en français où le déclassement du langage - écrire comme on parle - constitue une forme de régression choquante, que l'on a souvent, sous la pression du dogme de la convention d'un écrit " de qualité ", beaucoup de scrupules à transgresser.

97 Ainsi, sur des aménagements pourtant assez mineurs, il est parfois nécessaire pour le traducteur de se faire un peu violence.

Applications dans Camino Real - Mots déformés :

Chez Sancho, dans le Prologue: «Aw, naw », traduit par «Ah, nan », et chez Kilroy, Bloc 4 : «Nah, nah, nah! », «I say nah », « The nahs have it » traduits par « Nan, nan, nan! », « J'ai dit nan », « Les nan l'emportent! »; Chez A. Ratt et Kilroy, Bloc $4:$ « Yep » traduit par « Ouaip ».

Mots tronqués, élisions :

Chez Kilroy, Bloc 3 : « Keep y'r hands off me, y' dirty ole bag », traduit par " Garde tes 
sales pattes loin d' moi, espèce de vieux sac dégueulasse ", et "Now le' me think » traduit par «Maint'nant réfléchissons ».

Se faire ainsi violence, dépasser la convention et rester fidèle à ces graphies particulières sont un devoir pour le traducteur : en tant que telles elles sont des signes, il faut les respecter.

\section{Les mots en langue étrangère}

101 De nombreuses langues sont représentées dans Camino Real : outre l'anglais, on trouve du français, de l'espagnol, et de l'italien. Que faut-il en faire?

Le premier principe est de ne pas traduire ce qui est déjà de l'étranger dans le texte de départ, et qui n'est pas plus étranger au lecteur / spectateur français que ce n'était étranger à l'auteur / lecteur / spectateur de la version d'origine. En conséquence, il n'y a pas lieu de traduire les répliques ou expressions d'origines espagnole ou italienne.

Parfois cette position n'appelle pas de traitement particulier, parce que par exemple l'auteur lui-même donne la traduction des termes et expressions concernés :

Applications dans Camino Real - Ainsi dans le Prologue, où madrugada est conservé sans difficulté dans la mesure où Don Quichotte explicite lui-même le terme : «madrugada (...) qui lui aussi veut dire lever du jour ";

Ou encore, Bloc 2, où hermano est également conservé en l'état, car traduit par Jacques Casanova peu après : « That's the word for brother ».

Mais cette même position parfois ne manque pas de poser problème :

Applications dans Camino Real - Ainsi Bloc 2 encore, avec le chant de la Madrecita : «Rojo est el sol... Blanca esta la luna de miedo "; ici le spectateur risque fort de ne pas percevoir le sens de ces paroles, ce qui est toujours dommageable, mais que faire?

Le contournement pour le lecteur se fait via une note du Traducteur;

Le contournement pour le spectateur est autrement difficile, et peut passer par l'utilisation d'éléments de décor, d'accessoires, ou d'un jeu scénique particulier.

Ou encore, Bloc 4, avec ingreso libero (" entrée libre »), seulement traité, pour le lecteur, par un renvoi explicatif, la prise en charge du spectateur étant laissée aux bons soins du metteur en scène et des comédiens.

Il n'y a donc pas ici de solution complètement satisfaisante.

110 S'agissant de la question du respect de ce qu'on appelle l' " étrangéité » des mots, que faire des mots et expressions... en français dans le texte ? Comment reproduire cette étrangéité, qui ne sonne comme telle qu'aux yeux et aux oreilles des lecteurs / spectateurs... non francophones ? Faut-il traduire ces mots et expressions français... en anglais? Nous avons pris le parti de laisser en français le texte déjà en français dans le TD, d'identifier cela par des italiques et un renvoi, charge étant laissée là encore aux comédiens de faire passer cette subtilité aux spectateurs.

111 Une autre source de difficulté tient aux passages (en anglais) que l'auteur lui-même a traduits (ou transposés) du français et qu'il convient donc de retraduire en français. Bloc 4, le Clochard et le Baron donnent leur version de Padam Padam, la célèbre chanson interprétée par Edith Piaf : leur « Pa dam pa dam pa dam | Echoes the beat of my heart » correspond à une traduction de 1952 de « Il arrive en courant derrière moi » que l'on pourrait ici retraduire en un "C'est l'écho des batt'ments de mon cœur »... très éloigné 
du texte original ! Situation qui n'est pas sans rappeler Mark Twain qui, en 1875, et aux seules fins de démontrer à quel point une traduction pouvait s'éloigner de son texte d'origine - et le trahir -, avait retraduit en anglais, et publié, la première traduction française qui avait été donnée quelques années plus tôt de son conte sur la Jumping Frog of Calaveras County, retraduction qui avait peu à voir avec son texte original.

Dans un tel cas, faut-il alors retenir une retraduction en français de la traduction en anglais donnée par l'auteur, ou revenir directement au texte français d'origine? Toujours par souci de fidélité au texte de départ, nous avons choisi de nous appuyer sur le texte anglais, ce qui revient donc à donner une «nouvelle version » de la chanson en français.

Il existe également des mots que l'on choisit finalement de... ne pas traduire :

Applications dans Camino Real - Bloc 4, comment aborder le propos de Charlus, "The name of the Camino is not unreal », propos qu'il est difficile de traduire du fait de la nécessité de conserver intacte la résonnance avec le titre de la pièce?

La traduction proposée s'appuie sur une "réinjection" en anglais, permettant non seulement de ne pas perdre le lien avec le titre de la pièce, mais aussi de mettre le spectateur sur la voie du glissement de sens que l'auteur y a inséré, et enfin d'expliciter le jeu de mots de Charlus : « Le nom du Camino c'est Real, pas Unreal ».

Bloc 9 se trouve le cas intéressant d'une réplique en français dans le texte, mais immédiatement traduite par l'auteur : «Ici la Douane! Customs inspection here! » :

117 Le traducteur a donc choisi de laisser telle quelle cette réplique... bilingue d'origine.

118 Enfin, se pose la question des termes, noms et expressions anglais traduits par... un autre terme, nom ou expression anglais: le Funny paper du Bloc 12 ("A section or supplement of a newspaper containing comic strips", d'après thefreedictionary.com) devient ainsi dans la version française... « Comic strip », expression que le traducteur a estimé suffisamment assimilée par la langue parlée française.

119 Derrière tous ces exemples, une leçon se dessine en filigrane : le traducteur doit veiller à ne jamais se laisser enfermer dans des do's and don'ts, mais à toujours aborder la traduction comme une affaire de circonstance, de jugement, et de rythme.

\section{Jeux sémantiques et jeux de sonorités}

Les jeux de mots constituent l'un des domaines les plus compliqués de la traduction, en ce qu'il tend à rejoindre d'une certaine manière la poésie, avec des glissements de sens qu'il est souvent extrêmement difficile de reproduire fidèlement en français.

121 Application dans Camino Real - Bloc 6, comment traduire la réplique de Kilroy, «I been in countries where money was not legal tender. I mean it was legal but it wasn't tender ", réplique où Tennessee Williams glisse un jeu de mots entre le tender de « cours légal » et le tender de « tendre »?

Il faut dans un tel cas chercher dans la sphère sémantique de l'expression concernée, en l'espèce legal tender («cours légal»), un appui sur lequel construire un jeu de mots peu ou prou équivalent; ce qui a conduit le traducteur à proposer : "J'ai connu des pays où l'argent ce n'était pas la devise légale. Je veux dire, c'était légal, mais on n'en faisait pas pour autant une devise. » 
Les jeux de sonorités, assonances, allitérations, présentent également un niveau élevé de difficulté, quoique légèrement moindre peut-être (si l'on met de côté le «contreexemple » relevé précédemment avec le délicat « No one is gypped at the Gypsie's »). Mais l'exercice reste assez redoutable.

Application dans Camino Real - Bloc 4, comment traduire «I used to wonder. Now I simply wander ", en respectant la quasi-homophonie de wonder et wander?

Là encore, afin de rester le plus proche possible du texte de départ, il a paru nécessaire au traducteur de ne pas quitter la sphère sémantique d'origine, ce qui l'a conduit à opter pour « J'ai cherché à comprendre, je voulais que ça tourne rond. Maintenant, je me contente de tourner en rond». Et si l'image n'est pas exactement la même, la hiérarchie des effets - à parts égales, le sens et le jeu de sonorité - est respectée et l'impact sur le lecteur / spectateur correctement traduit.

\section{Les figures de style et l'« enrichissement excessif »}

Le traducteur peut parfois ressentir la tentation - ou la nécessité s'imposer à lui d'introduire une figure de style là où le texte de départ n'en comporte pas.

Application dans Camino Real - Bloc 8, deux approches peuvent s'envisager pour traduire les propos de Lord Byron, «I can look up at broken columns on the crest of a hill »; une première option consiste à traduire "à plat ", en suivant littéralement le sens de la phrase: «Je pourrai contempler les colonnes brisées sur la crête de la montagne »; dans une seconde option, il est possible d'animer ce qui dans le texte de départ n'est qu'un élément inanimé, "la montagne»: "Je pourrai élever mon regard vers ces colonnes brisées dont la montagne hérisse sa crête ».

Le traducteur doit-il rester littéral ou peut-il s'autoriser à introduire une dimension (animé vs inanimé) et une image (le «hérissement» de la crête), toutes deux inexistantes dans le texte de départ? C'est là une grande question; très vraisemblablement, le juge de paix est dans le rythme (comme souvent), qui doit « coller » aux circonstances, c'est-à-dire à la tonalité originale, à la couleur du passage, et aux conditions dans lesquelles se trouve le personnage.

Or en l'espèce on observe qu'il existe bel et bien une rythmique dans le texte anglais (deux binaires : bro $\mid$ ken co $\mid$ lumns, suivies de deux ternaires : on $\mid$ the $\mid$ crest, of $\mid$ the $\mid$ hill), qui véhicule une sorte de solennité dont est dépourvue la traduction "à plat ", tandis que l'option alternative, substituant à cette rythmique d'origine une image vigoureuse mise en valeur par la double allitération en " $\mathrm{r}$ » et en « $\mathrm{s}$ ", donne au texte d'arrivée une belle force sonore et évocatrice; dans cette seconde option, l'impact sur le lecteur / spectateur - un mix de sens et de musique - semble correctement traduit.

Mais ne risque-t-on pas ici de tomber dans ce défaut grave que l'on peut désigner comme l'«enrichissement abusif ", qui revient à trahir le texte en le chargeant de dimensions, images et messages absents de la version d'origine? Ce ne semble pas être le cas dans notre exemple, tant cet enrichissement reste ici mesuré et discret. Mais il n'empêche : ce défaut doit être combattu avec la dernière vigueur : emporté par son élan, grisé par les phrases qui s'alignent et se déroulent, prenant parfois pour siennes des qualités qui ne sont que de l'auteur, le traducteur se sent pousser des ailes et risque de se laisser entraîner, à moins que ce ne soit pour des raisons peut-être de meilleure accroche commerciale ou autres encore, comme dans les deux exemples suivants (hors 
Camino Real), où le même défaut s'observe sous les jours différents de l'enrichissement abusif, avec "A moveable feast ", titre d'Ernest Hemingway, devenu dans sa traduction française, «Paris est une fête " ${ }^{5}$ et de l'« appauvrissement » excessif, avec « Their Eyes Were Watching God », titre de Zora Neale Hurston, devenu le très (trop) simple, «Une femme noire $»^{6}$.

\section{Gestion des « béquilles » (ce qui permet de « faire tenir debout » le texte)} dire, au texte.

Réorientation : fait entendre »;

Ponctuation :

Les « béquilles » sont tout ce qui permet de faire tenir debout la traduction : il en va ains de tout ce que le traducteur fait pour fluidifier, structurer, clarifier le texte... en coupant, en ajoutant, en réorientant.

Le risque des «béquilles ", c'est qu'elles sont souvent... très visibles! Elles sont donc à manier avec prudence et modération.

Applications dans Camino Real - Dépronominalisation:

Bloc 8, échangeant avec Casanova sur la vocation du poète, Byron déclare : «A poet's vocation, which used to be my vocation, is to influence the heart in a gentler fashion than you have made your mark on that loaf of bread. He ought to purify it and lift it above its ordinary level ». Une traduction littérale, respectant l'emploi d'origine des pronoms, conduit en français à une multiplication de ces derniers qui, conjuguée à l'éloignement entre les pronoms he et it et les noms poet et heart auxquels ils renvoient, nuit à l'immédiateté du sens et rend la phrase ambiguë, phrase dans laquelle le lecteur / spectateur se perd. D'où la tentation - la nécessité, même, ici - de « dépronominaliser » le texte pour rendre sens et clarté à la phrase : «Sa vocation, ce devrait être de le purifier, ce cœur, et de l'élever... » ;

Cette technique toutefois peut vite devenir une solution de facilité, qui présente le défaut majeur d'alourdir et de donner un tour quelque peu «scolaire", si l'on peut

Au tout début du Prologue, le lecteur / spectateur apprend que « There is a loud singing of wind », construction existentielle très commune en français parlé, mais assez lourde en français écrit, que le traducteur pourra préférer réorienter en « Un vent puissant se

Il s'agit là d'une technique sans intérêt particulier, relevant véritablement de la "mécanique de routine » de la langue - même si en d'autres circonstances il peut y avoir là de vrais enjeux de sens et de tonalité.

Il s'agit ici d'une mention "pour mémoire » car... il n'y a pas dans cette traduction de Camino Real d'exemple de telles «béquilles » liées à la ponctuation; c'est un glissement qu'il faut là encore résolument combattre : l'ossature de la phrase originale doit être respectée, le traducteur n'a pas le droit de remplacer un ligament par un os ni même un cartilage... Les textes de Tennessee Williams sont assez peu sujets à de tels traitements, mais ceux-ci peuvent être très dommageables à des textes d'auteurs tels que Virginia Woolf, dont les immenses, profondes et onduleuses phrases pourraient être mutilées si 
un traducteur indélicat, ou pressé par le temps, se laissait aller à «simplifier » et à remplacer virgules et points-virgules par des points.

\section{Conclusion}


qu'il servira honnêtement et l'œuvre, et son auteur, pour le plus grand profit de la littérature et des lecteurs.

Fig 3

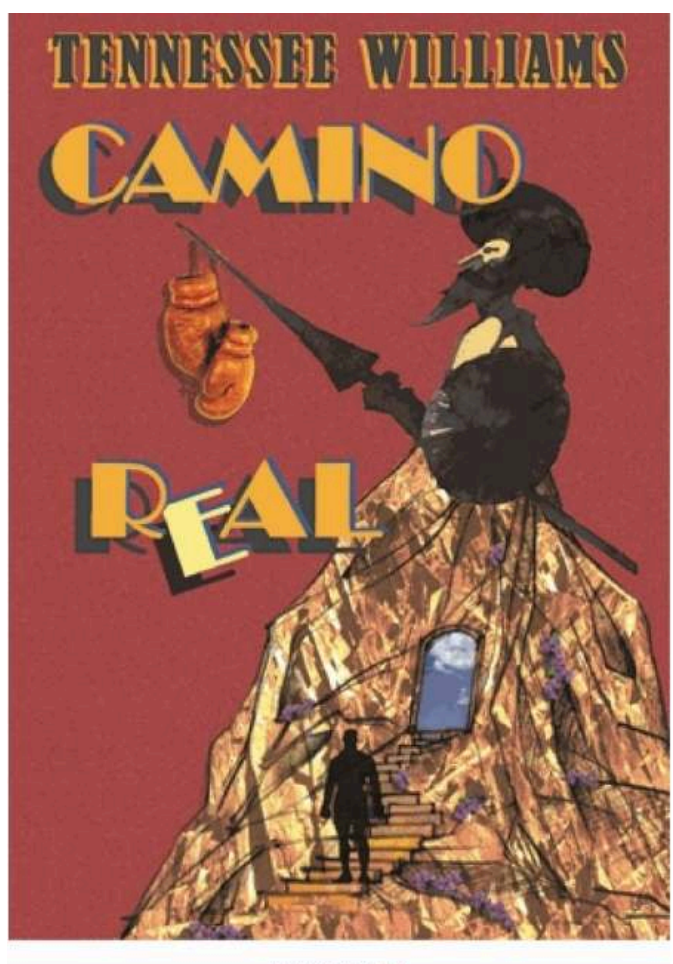

ANTIGONE14

Camino Real, Tennessee Williams, Trad. Bertrand Augier

Crédit : Antigone14

\section{NOTES}

1. Eau-forte: "Une planche de métal (fer ou cuivre) est recouverte sur ses deux faces d'une fine couche de vernis destinée à la protéger de la morsure de l'acide. À l'aide d'une pointe dure, le graveur entaille le vernis selon le tracé du dessin qu'il veut obtenir. Il fait ainsi apparaître, par endroits, le métal débarrassé de sa couche protectrice. Ce sont ces parties du métal dénudé qui seront attaquées, lorsque le graveur plongera la plaque dans son bain d'eau-forte. L'action de celle-ci jugée suffisante, le graveur sort la plaque, la rince à l'eau claire, puis enlève le vernis protecteur, découvrant ainsi toute la surface de la planche, qui présente des creux aux endroits où l'acide a agi. Selon le temps d'immersion, la morsure par acide est plus ou moins profonde et permet d'obtenir un trait plus ou moins marqué lors du tirage. L'opération dans son ensemble peut être renouvelée autant de fois que le graveur le juge nécessaire ", extrait de l'ouvrage Larousse " Dictionnaire de la peinture ", https://www.larousse.fr/encyclopedie/peinture/eauforte/152018 
2. Le "théâtre plastique» de Tennessee Williams: du "langage de la vision" à "l'écriture organique", thèse de doctorat en littérature américaine soutenue publiquement le 14 novembre 2014 par Sophie Maruéjouls-Koch, Université de Lorraine.

3. Référence au célèbre graffiti Kilroy was here ("Kilroy était là ») apparu de manière assez mystérieuse durant la bataille de Normandie et que les GI's américains se sont par la suite ingénié à répéter en de multiples lieux.

4. Lettre à Lawrence Langner, n.d., Yale, citée in The Selected Letters of Tennessee Williams, vol. 1, 1920-1945, edited by Albert J. Devlin and Nancy M. Tischler, The University of the South, 2000, p. 277.

5. Hemingway, Ernest, Paris est une fête, traduit de l'anglais par Marc Saporta, Paris, Gallimard, 1964.

6. Hurston, Zora Neale, Une femme noire, traduit de l'anglais par Françoise Brodsky, Editions de l'Aube, 1998.

\section{RÉSUMÉS}

Ce texte est la retranscription de l'intervention de Bertrand Augier lors du symposium ACT (Anglophone Contemporary Theatre - Laboratoire CAS/Univ. Toulouse Jean Jaurès ; dir. Mariane Drugeon, Emeline Jouve, Sophie Maruejouls) du 11 février à la Cave Poésie (Toulouse). Le traducteur revient sur son travail de traduction de la pièce Camino Real de l'auteur américain Tennessee Williams.

This text is a transcription of Bertrand Augier's paper at the ACT symposium (Anglophone Contemporary Theatre - Laboratoire CAS/Univ. Toulouse Jean Jaurès ; dir. Mariane Drugeon, Emeline Jouve, Sophie Maruejouls) on February, 11th, 2020 at the Cave Poésie playhouse (Toulouse). The translator comes back on his translation of the play Camino Real by the American playwright Tennessee Williams.

\section{INDEX}

Mots-clés : traduction, théâtre, théâtre plastique, Tennessee Williams

Keywords : translation, theatre, plastic theatre, Tennessee Williams

Thèmes : Theater

\section{AUTEUR}

BERTRAND AUGIER

Traducteur et éditeur

ANTIGONE14 Éditions

bertrand.augier@antigone14editions.com 